\title{
A NEW INTERFACE
}

\author{
SEAN CLARK
}

The means by which humanity communicates has seen great change over the last two millennia shaping the many ways we share knowledge today. Before the written word, oral traditions allowed for communication locally. If compelling enough these vocalised thoughts would move across time through retelling and fresh interpretation. Trained storytellers and singers enabled this early aural interface.

As the Roman Catholic Church grew across Europe, knowledge began to centralise. Monks labored to transcribe the Bible and other religious works while defining the page structure and layout of the written word. They created beautiful works of art through their illumination and helped to bring the written word and a new mode of knowledge sharing into the lives of those who could afford it. Unfortunately, the heavy labour of transcription meant that only a small quantity of books could be made each year. Then, in the middle of the 15th century, Johannes Gutenberg's invention of the printing press formed the defining moment in Europe's search for a better mass communication interface. An invention still important today, it made quick copying and mass dissemination of information possible. It also helped to standardise page structure and typography making it possible for more people to read and comprehend the text. The printed word remains the most trusted source in today's Information Age. The connected digital environment has again broadened access and added a new element, interactivity, into the formerly static page. However, digital media has yet to see its full potential as it remains tied to the traditions and axioms of print design that came before it. A new interface, free of the strictures of print design, could have an impact not unlike Gutenberg's invention of the printing press.

The term interface may have had its origins in the sciences, but today it is generally accepted as a term used to describe how a person interacts with a computer. Merriam-Webster defines it as "a system that is used for operating a computer: a system that controls the way information is shown to a computer user and the way the user is able to work with the computer" and "the means by which interaction or communication is achieved at an interface". Further, it has come to simply mean the direct interaction between people or between a person and a device when transferring information. Through this lens we can look back across history and view the printed page, the illuminated manuscript, and the conversation of the early illiterate masses as interfaces. Technology has played a large role in humanities progression through these modes of communication. Even the printed page required advances in the production of vellum, parchment, and finally paper to be fully effective. A more concise definition might look something like this: a mode of expressing or displaying thought in an organized manner through aural, visual, or interactive means in order to share knowledge. 


\section{COMMUNICATION INTERFACES}

Oral tradition or the expression of thought by a storyteller or singer to localised communities of listeners is in itself both a form of technology and interface. Albert Bates Lord defined this tradition, "for any individual singer the tradition consists of all the performances of all the songs of all the singers he has ever heard. All the singers encompasses the worst, the best, and all in between. ... A tradition is dynamic and ongoing" (Lord, 1995, p. 3). This trained individual was called a Filí in traditional Irish culture (Nagy, 1986). Before the spread of the Latin alphabet and literate populations, he had linguistic methodologies and memory devices he could use within his speech to help listeners understand and memorise his stories; not so much as changing them, but simply making them more relevant to the current listeners. A modern day example can be heard in the performance of traditional jazz. These jazz musicians learn not solely by reading, but by listening to other musicians. They improvise within their own performance to the benefit of the audience listening at that moment while maintaining the themes and ideas from previous renditions of the same piece. A recording of this piece of music would only capture the musicians' performance at that moment, creating a static snapshot, though variations on the same piece exist across time.

When oral "genres first appeared in writing, their metric base, their poetic and compositional devices, were already fully developed and none of them could have been invented by any person at any one time" (Lord, 1995, p. 1). Lord argues that early scribes used the structure and devices of the storytellers and singers that came before them. He also theorised that the discrepancies between written versions of early stories was due not to errors in the scribes' transcriptions, but to them hearing different versions. In committing these stories to the permanence of the page, the scribes stripped them of their dynamism and lent permanence to the singular versions that they had heard. The spread of the Latin alphabet along with Christianity across Europe brought a major new communication tool into the lives of the storytellers. Nowhere was this more evident than in Ireland where oral tradition and the written word lived, seemingly equally, side by side. In a talk given by Joseph Nagy (1988), he spoke of evidence pointing to close connections being formed between the old oral traditions of the Fili and the new monastic class who were well-learned in Latin and the literature of late classical Christianity as Ireland moved out of the 5th century.

In transcribing the Bible and other religious texts, scribes, generally monks at this time, worked with and as artists. They left room on the page for initial caps, rubrics, and later for miniatures that grew ever more elegant and complex. They created margins around the text and sometimes commented within them. Their art progressed into illumination which required the use of gold and silver to literally light up the page. This interface progressed from the oral tradition before it by using the visual sense to pull the reader in and hold their gaze (Bradley, 2006, p. 32). Interest in the ownership of these illuminated books spread from ecclesiastic to the secular. The wealth and royalty of Europe began requesting books on a variety of subjects to fill their private libraries (Jones, 2000).

As a new industry of non-religious publishers provided greater access to information, literacy began to spread among the common peoples of Europe. The control of published information moved from a closed system to one that was more broadly open. It also reawakened the ability of non-religious oral traditions to spread through written word. As an example, William Shakespeare wrote his plays to be seen and heard, but the printed versions have had a large impact on the literary world that followed. Gutenberg's greatest achievement was to bring information access to the masses. The printing press created a greater economical means for spreading knowledge versus the scribes and illuminators it ultimately replaced. Through the relative openness of the media and the permanence of this new interface, the printed page became the most trusted, if easiest, to access sources for knowledge. 
New forms of print were created such as newspapers, magazines, and pamphlets. The mechanically printed page retained the layouts formulated by the monks and secular scribes by continuing to use margins, initial caps, white space for hand drawn illustrations, and carrying on the practice of using red ink for initial paragraphs. Gutenberg's printing press ultimately served to broaden and speed up the average person's access to information while maintaining rules of structure and layout that were recognisable across all forms of print.

Today's printed page, especially in book form, is still the most trusted source of information. However, digital media and the Internet as humanities latest innovations in transmitting knowledge are slowly replacing the printed page through convenience and perceived cost reductions as the primary source for knowledge. Born with tremendous potential, the Internet promised to share more information than any medium before it through an interface that can support all forms of media and in an interactive way not previously possible. The Internet and its related technologies connect people across the globe in instantaneous communication, sharing information as we never could before. A young student in the United States and another in Estonia can quickly and effortlessly share local experiences across differing cultures, geographies, and even languages. Digital media and the Internet are now in a state of transition between the confines of print norms and the promise of an all inclusive and sharing medium that can truly revolutionise the way we find and share knowledge.

\section{TOWARDS A NEW INTERFACE}

Today's digital interface has several barriers that must be overcome for it to achieve its full potential. The vast majority of websites and apps are formatted to look, or simulate, their printed counterparts. They often make use of only a fraction of the screen which has an aspect ratio ranging from 4:3 to 16:10. This has become more apparent over the last few years as technological advances have migrated the screen to wider aspect ratios, whether in standalone monitors, laptops, tablets, or smartphones. The latter wreaks havoc on typical web design because of their small size and the user's ability to change the orientation between portrait and landscape at will. On the other hand, the tactile and on-screen interfaces of smart phones may help to push digital media beyond the confines of print if designed for properly. Along with these, a clutter of information often distracts from the main content or most important element of a page.

Another challenge for digital media in this transitional period is tied to its place within the computing world. Over the last couple years the design community has argued over the use of skeuomorphs in website and app design. These metaphorical and representative uses of imagery help a user connect a digital concept to a real world object; take the image of a file folder used to represent a place where digital files are stored on a computer as an example. The larger argument centres around the use of real world textures, three-dimensional fakery, and the representation of everyday objects within the flat display space of the screen based interface, highlighted in the much cheered changes in the look of Apple's iOS7 interface. However misrepresented, this argument is over aesthetics and design choice. The old iOS interface may have been kitschy, but it was also very popular and making big changes, as Apple did, can confuse users.
Metaphors will always find a place in digital media design. Their appearance, whether flat, three-dimensional, or somewhere in between, is up to the designer while likely being influenced by current trends. Most importantly these visual displays must always make content the first priority and make sure that the user has easy access. 


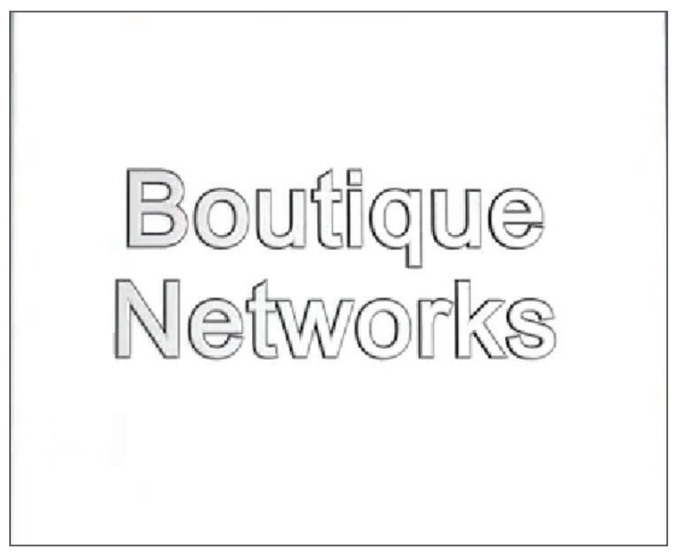

Figure 1: Boutique Networks.

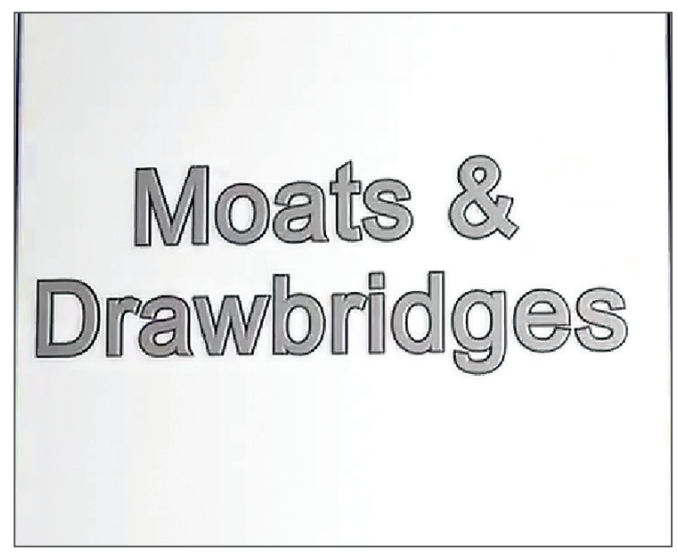

Figure 2: Moats and Drawbridges.

From the turnable pages of books, to economics and social media, access may be the biggest road block to the evolution of the connected digital media interface. The Internet Society, a non-profit organisation formed in 1992 to promote standards and open access on the Internet, has some concerns about the future of connected devices. Through a scenario called the "Boutique Network Scenario" in Figure 1, they show the possibility of the Internet becoming segmented in a future where everyone connects to a localised intranet and have no access to content outside of it. In another example called "Moats and Drawbridges", Figure 2, they show a future in which governments and corporations work together to wall off data an charge large fees to move through these walls while also removing creative control from the content developers (InternetSocietyVideo, 2010). These sorts of controls and centralised powers would be more akin to the publishing industry that rose with the invention of the printing press constricting control of content. Under these scenarios a few big players will control everything and digital media will not be able to reach its full potential. The individual who has leveraged the openness of the Internet to self publish will no longer exist. Less competition will also reduce access through higher costs to the user. The possibilities here are all the more real given current global events and concerns about security. Governments may push to lock the virtual gates of their networks and only allow traffic within their own borders, thus destroying the global community and sharing of knowledge that the Internet has fostered since its beginnings.

In efforts to better monetise digital media, search engines such as Google and social networks including Facebook have worked to personalise content displayed to each individual user. In 2011, Eli Pariser coined the term "filter bubble", Figure 3, to explain how this works to narrow the information displayed to a user by showing only what they are most likely to want to view (Pariser, 2011). The user no longer receives information counter to or outside of what they have unwittingly told the provider they prefer to see. While these large Internet based companies may mean well they are working counter to societies views of what the internet should be. Narrowing visible content removes an advantage of connected digital media in allowing a user to quickly find information across an entire field of possibilities from any location and in the long term may affect their own world view. Three computer scientists now say they can reverse this trend with an algorithm that uses the similarities between users in one subject area to show them counterviews in another subject. Their research has shown that users actually prefer to have access to information counter to their own views and that users are more likely to stick around when the content served to them is more fully rounded (MIT Technology Review, 2013). For digital firms concerned about their bottom line and maintaining users, this should be good news and impetus to maintain fully rounded content for each individual user instead of placing them within a more restrictive filter bubble. 


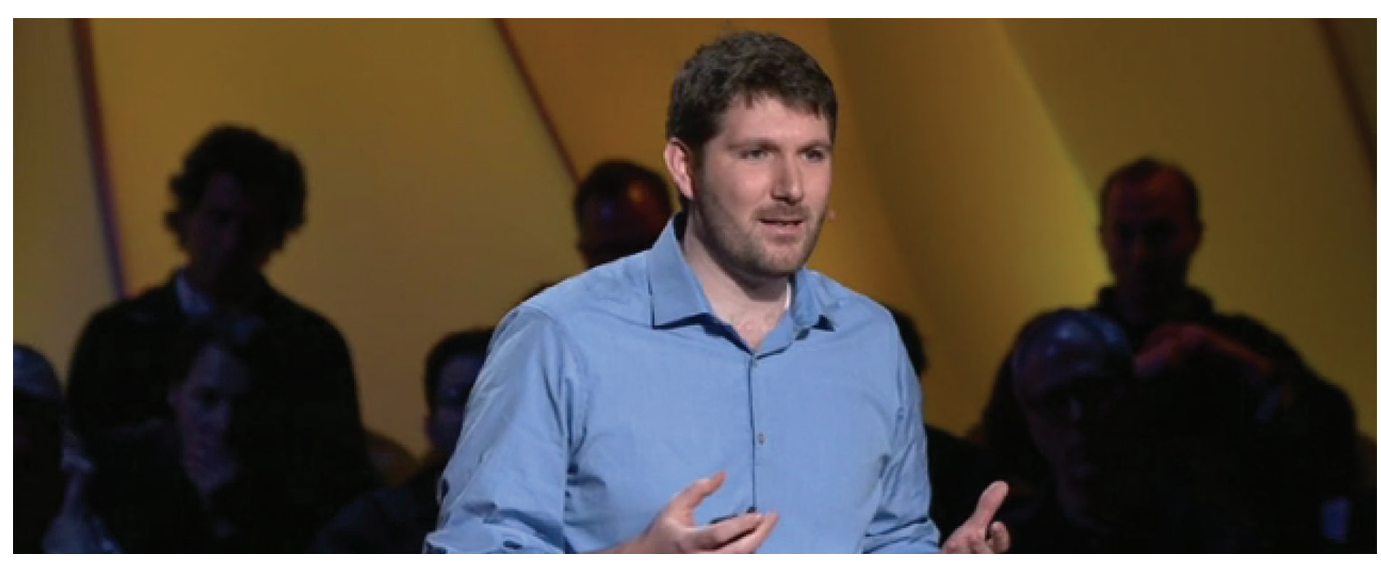

Figure 3: Eli Pariser, Beware Online "Filter Bubbles".

Designers must consider content as top priority and place it highest in their visual hierarchy. Whether designing for typographic or other forms of content, the user's intent should come first. The page structure and layout created by the early writers of manuscripts lived on through Gutenberg's printing press and moveable type. However, typography is struggling in the digitally connected domain where the form factor of the displaying device varies greatly and too much is being placed on the screen to grab the user's attention. As standards progress and designers move towards responsive designs, typography on the Internet is coming of age. Digital typography also has an advantage over the printed page in its interactive ability. Many websites and digital devices now allow for the scaling of text and changing of foreground and background colours by the user to better suite their environment and needs. This helps the content remain at the top of the hierarchy and engages the user in continued use. Responsive design as a whole can keep content up front no matter the size or orientation of the display. This flexibility is where digital has its advantages over print.

Connected digital media has several advantages than can elevate the screen above the printed page. Both content and user can be anywhere in the world separated by geography, culture, and political boundaries. The user can access the information almost instantaneously at any time from the moment it appears on the internet. That content is also fluid and flexible. The device used to display it can vary in shape, size and functionality. It can be displayed on the latest highest end smartphone or on a low cost "dumb" phone that is connected and at the very least displays simple html pages. The interface served up to the two phones may differ, but the content can remain the same removing barriers to those with fewer and lower tech choices in device. It can be updated at any time to reflect current knowledge and can link to and from other pertinent information. The greatest advantage that digital media has over print is in its mode as an interactive medium. A well designed and connected digital interface can engage users like no other medium can in sharing knowledge. It can guide users through content while also allowing them to navigate freely to discover the wealth of information at their fingertips.

It is in the Internet's ability to store and share all forms of media that this can be best accomplished. When the interface combines audio with imagery and animation, or the occasional video, it can become more engaging to a broader audience than print. That audience might even learn more through the multi-pronged presentation of information. The Internet as a malleable medium can look very different on one screen versus another while still conveying the same information. Today we think in terms of a rectangular screen, but soon it could be something very different. Smartphones can create new environments and add to the ones we know through a concept known as augmented reality. When a user points his smartphone at the 


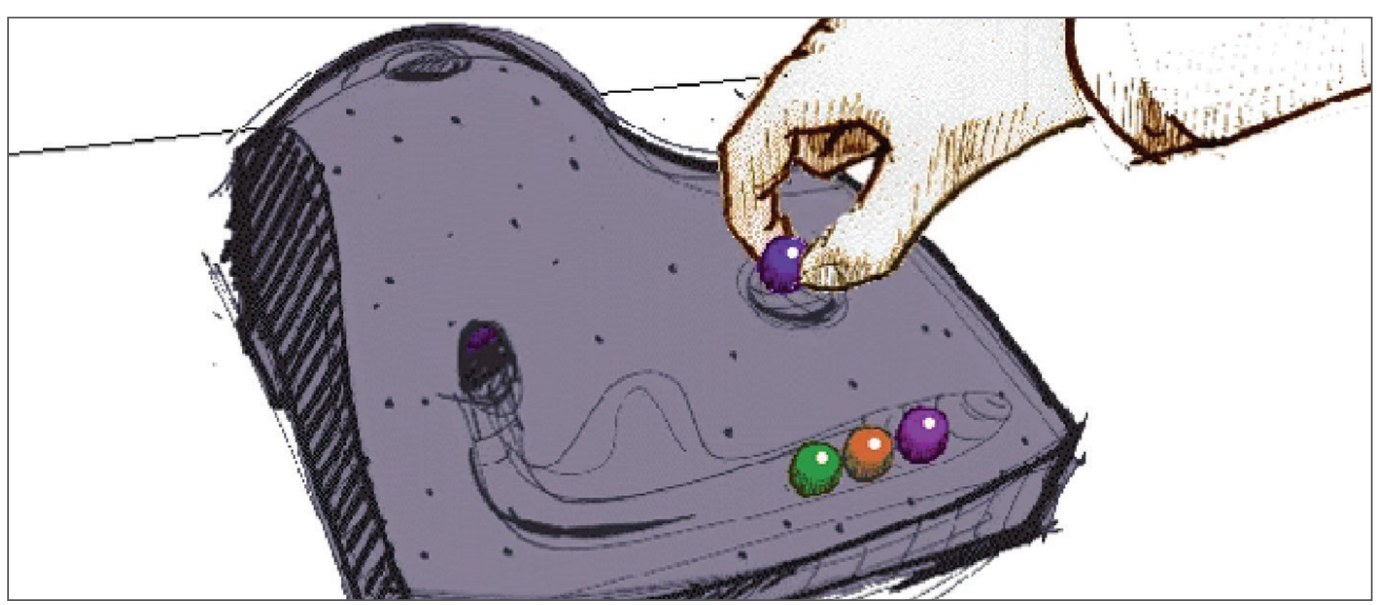

Figure 4: Durrell Bishop, "Marble Answer Machine".

real world, it can use its various sensors to place virtual objects within it for the user, even while moving around, creating a whole new experience. The rectangular screen then appears more as a window on the world to look through rather than directly at. The next step might be something like Google Glass, noncorrective eye wear which will allow the users to look directly at the world around them while simultaneously seeing location relevant information right at eye level. However, there is a counter argument for interfaces that are more physical in their interactions with digital media. An example is given in Durrell Bishop's 1992 prototype of an answer machine, Figure 4, uses different coloured marbles to represent messages left by people calling when he is away. These marbles can be placed back into the machine to play or erase the message or directly on the phone to initiate a call. They can also be placed elsewhere as a reminder for later use (Do, 2011).

\section{THE LOOK OF A NEW INTERFACE}

New ways are needed in looking at how information is stored, searched for, gathered, and displayed on the Internet and digital devices. Ethan Marcotte, in his "The Map Is Not the Territory" talk (2013), Figure 5, argues that we are still using a vocabulary meant for print when discussing knowledge shared over digital media. He believes that we need a new definition of beauty in web design and that it should include ease of access as a high priority. As we move through this transitional period, Bret Victor argues that we are too focused on the screen as an interface, that there is nothing natural about it. He argues for a new user interface that truly allows us to use our hands (2011). The smooth glass screen is perhaps not the only answer to connecting users to the dynamic information that lives on the Internet.

The trusted sources of knowledge is moving from the large publishers and media corporations that have controlled information since Gutenberg's time and back to the common person. Through this shift the Internet has taken on a aural sense. Through blogs and social media site such as Twitter the everyday person posts their thoughts, often with little editing, and while the Internet lends more permanence to these digital bits, they are often treated just as aural populations of our past treated the non written word. We are returning to information sharing as a social medium rather than a professional medium controlled by a few big players. Communication in an aural sense, store as digital bits, has expanded beyond the local community. The difference today is that we as individuals can share on a global scale. While the interface that will bring connected digital media into its own does not yet exist much has been said about what it may look, sound, and feel like. 


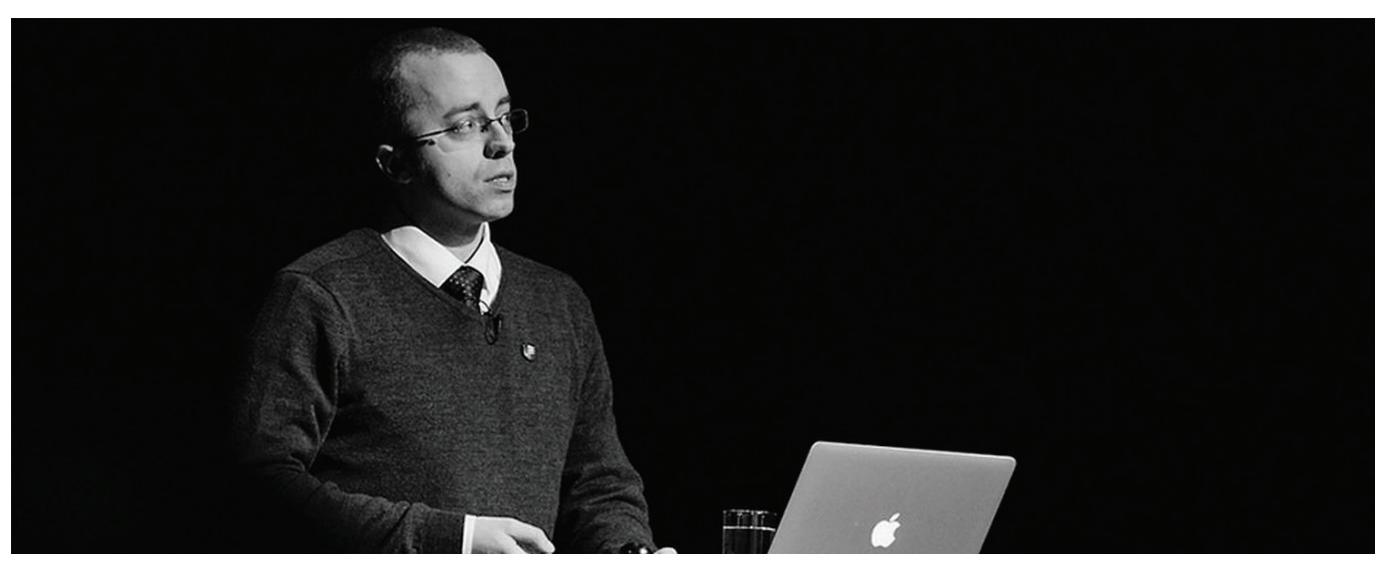

Figure 5: Ethan Marcotte, "The Map is Not the Territory".

In a talk given by Vinton Cerf, Figure 6, he mentions a comment made by one of his colleagues, "one hundred years from now people are going to say, 'did you know that one-hundred years ago we didn't have books that talked to each other?"' (2009). This is an interesting idea that someday soon books will be able to communicate and update dynamically on their own. But as Cerf mentions in his talk, this creates problems. How does the user know where that bit of information they found earlier has moved to or has it changed totally? This interfacing of information between different sources will create a new challenge in the interface to users. If we look at digital media in the print or typographic form only, what is really needed is simplicity.

Returning to Ethan Marcotte's talk, designers should also consider the global user. Many people do not have a flashy, latest off the production line smartphone. In many markets people are still using simple text based phones. If the design goal is to make content beautiful by making it accessible to the simplest of devices, we can reach more people. In doing so, more users would benefit from the spread of knowledge (2013). If we take this a step further perhaps the interface can also be improved by where information is pulled. If all information is placed in the cloud, a massive network of databases, or multiple and interoperable cloud systems it will make for better information sharing if data about a single subject can be pulled from multiple sources. To illustrate, a Bible scholar doing a single search on a passage would be able to see various versions and translations of that single passage. He would not have to look at multiple web pages or search libraries and collections for multiple books. Instead, he would see within a single unified interface all of these versions. The interface pulling these bits of information from various sources into a single interface that allows the scholar to quickly go back and forth between them and pull them all together for a better understanding of the passage and how it may have changed over time and through translation.

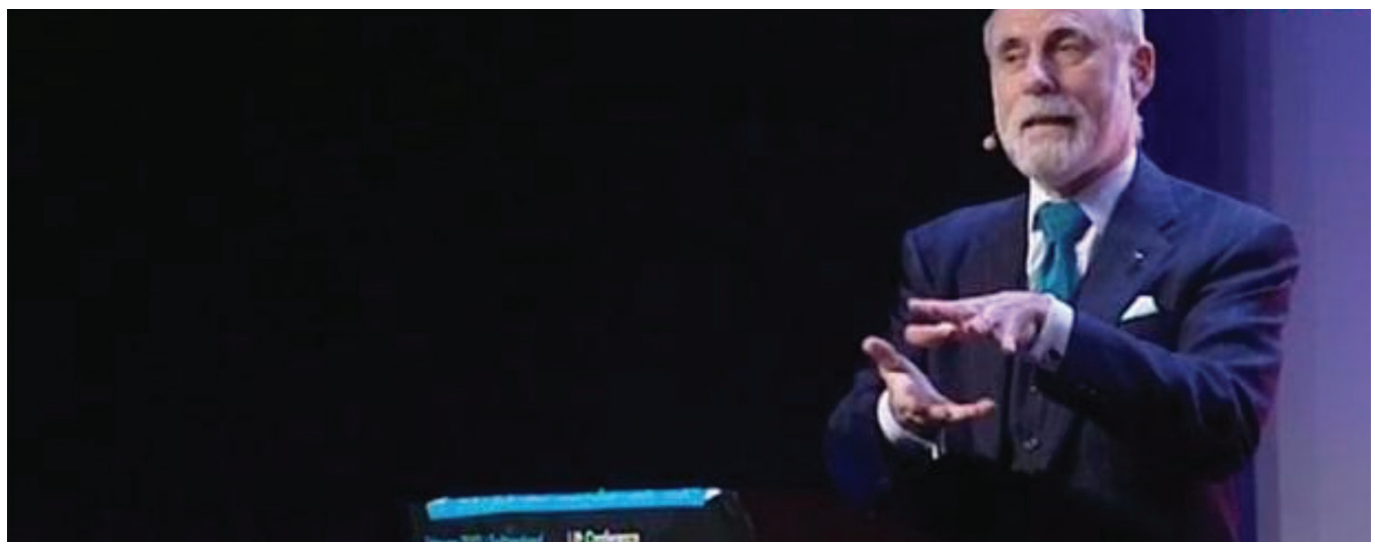

Figure 6: Vinton Cerf, "From the Jurassic Era". 


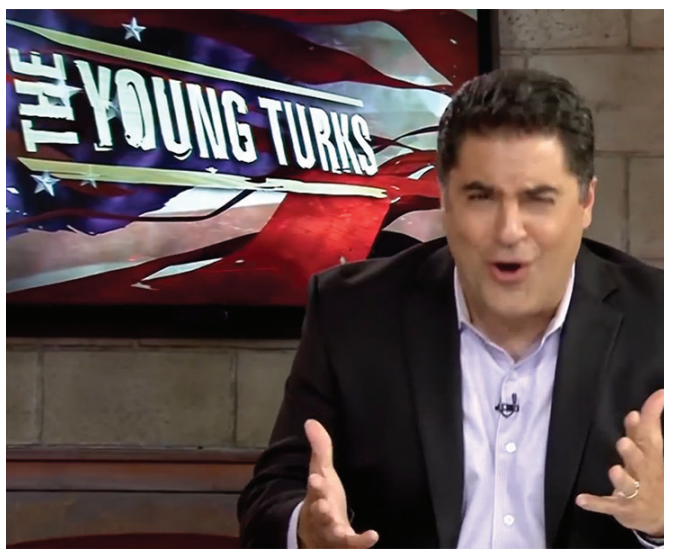

Figure 7: Cenk Uygur of The Young Turks.

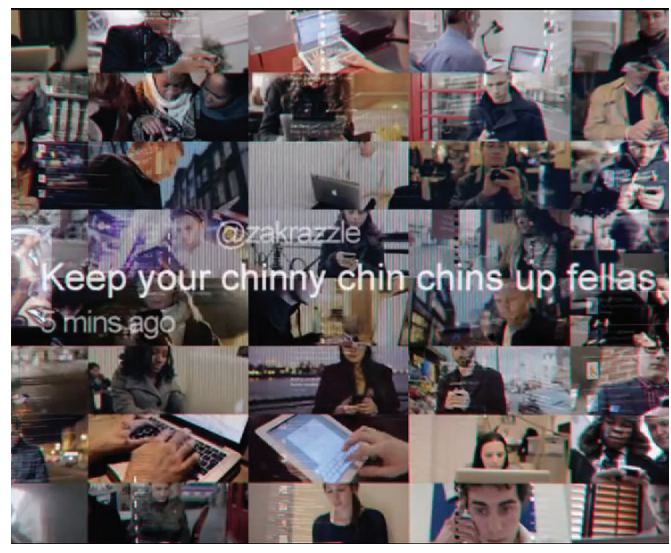

Figure 8: The Guardian, "Three Little Pigs" Ad.

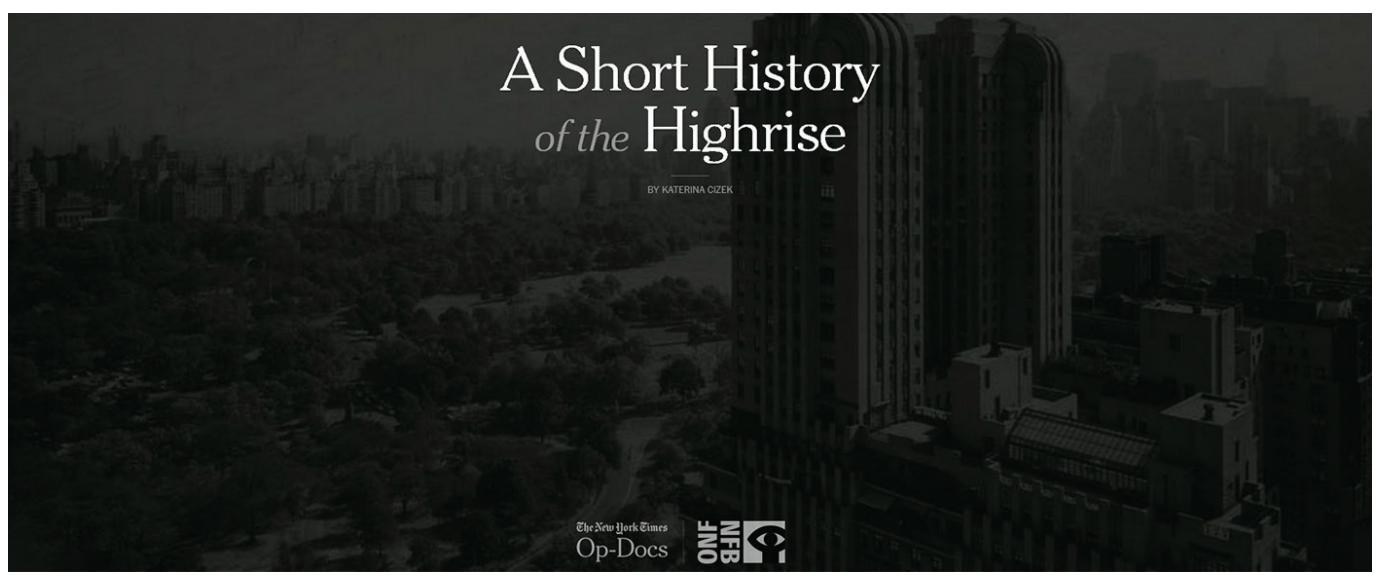

Figure 9: The New York Times, "A Short History of the Highrise".

Social media has reintroduced society to the aural mode of communication that existed before the written word. Except this time it is happening on a global scale and with more permanence than the purely vocal word. This is happening through short phrases on sites like Twitter and videos on sites like Youtube and Vimeo. In the process these posts from everyday people and independent groups are displacing the traditional news media. Figure 7 shows a still of Cenk Uygur of The Young Turks, an independent news organisation that broadcasts on their own Youtube channel and claims to be the most watched news on the Internet. Figure 8 is a still from The Guardian's ad for their open journalism project. The ad depicts how social media would react and how The Guardian would report the story if the three little pigs existed today and were blamed for the apparent murder of the big bad wolf. In their new initiative The Guardian is harnessing the views and perspective of the everyday reader to help them report the news. The New York Times has begun to move their print media towards a more interactive and digital media friendly format. In examples like "A Short History of the Highrise", Figure 9, they combine text, images, audio, video, and animation all into a near seamless interface in which the user can push and pull at the elements and move forward and backward through the story at will. The user can decide whether to watch, listen, and interact with only some portions of the story or enjoy it in its entirety. In doing so they are moving traditional media towards a model that supports and utilises the digital interface that connects people to knowledge and information while acknowledging the renewed aural sense that instant and global connectedness has given society. 


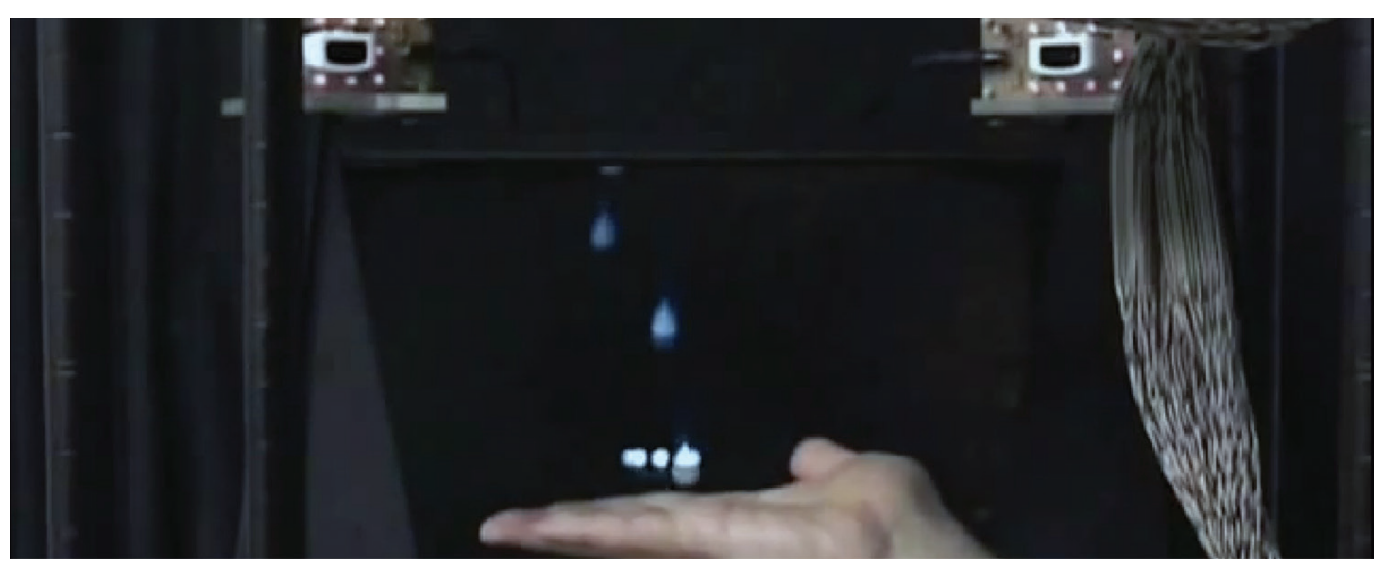

Figure 10: Shinoa Lab, "Touchable Holography".

In an essay by Frank Chimero he writes that "web and interaction design are just as much children of filmmaking as they are of graphic design" and that screens do not care what content is being displayed they just want it to move; he calls this movement "flux" and defines it as covering everything from animation and user customisation to the responsiveness of the site itself (2013). If we take Shakespeare's plays as an example, the best way to experience them is to see them performed. However, to understand and truly appreciate them requires study. An interface that takes advantage of flux and can operate in an open environment to pull video from different performances, text from different folios, and scholarly knowledge together will enable greater understanding and appreciation. Through intertwining the written word with audio and video and showing them in context, the user will achieve this goal. However the interface must go beyond this, it must adapt to the device in hand. If the design is successful, it will not only respond to screen size but scale to show only the content the device is capable of while maintaining a clean and user friendly appearance. The interface should be able to construct a full multimedia experience, but at the same time leave no device behind or cause the content to appear broken due to lack of capability.

Haptic feedback, a feature missing from stock iOS devices, but available on most Android devices, enables a short subtle vibration every time the user tabs a key on the virtual keyboard of their smartphone. This vibration acts as feedback that immediately alerts the user through touch that they pressed a key. It is similar to the audible beep of older phones with physical buttons but provides more direct feedback that is only noticed by the user. Feedback is essential to human understanding especially where the sense of touch is concerned. The vibration on a key press is only a small example; feedback is largely missing from the interfaces of our digital world.

Returning to Bret Victor's essay on the future of interaction design, he calls the touchable glass interface of current devices "Pictures Under Glass" and writes that they "sacrifice all the tactile richness of working with our hands, offering instead a hokey visual facade" (2011). The future of digital design may be less digital. Engaging the user more deeply may require more feedback, more physical interaction than can be had with smooth glass surfaces. At SIGGRAPH 2009 a group of researchers from Shinoda Lab showcased a video, Figure 10, in which they demonstrated that users can interact with holograms through the use of motion tracking cameras and focused ultrasound waves that create force and allow for tactile feedback. The user in the video can feel the virtual raindrops hitting their hand. This of course is a very rough technology that is barely into its infancy. However, in the future it may be a more practical means for interacting with digital media than the example given in the last section of the marble answer machine. 
As an example, imagine sitting in your future living room and you are about to assemble a piece of furniture. Many people today find printed assembly instructions hard to follow. What if in that future living room you could go to a hologram display in which you opened up a virtual manual. The tactile hologram would allow you to manipulate the various pieces and maybe even practice assembling them before you sit back down to assemble the real furniture. The digital interface of the future may be more familiar, more tactile and more inviting that the shiny glass screens we interact with today.

\section{IMPACT OF A NEW INTERFACE AND CONCLUSION}

It is unlikely that a single interface will elevate digital media above the printed form. A mix of different interfaces providing access to our digital world is advantageous. The seven billion people now living in varying cultures, topographies, and means do not all learn alike, they do not use the exact same digital devices, and indeed they each have preferences of their own if not simply differences in options based on geographic location. New designs that harness all that digital media has to offer from textual, visual, audio, and mixed sources will allow users to customise their experience to their own needs and learning style. The return to an aural sense of communication spurred by social media will force large media and news corporations to adapt or disappear. The Guardian's open journalism project is an example of this shift and The New York Times has begun to show that old media is willing to invest in redefining how they share the news instead of simply pasting what they print into digital media that mimics the paper medium. A new economic model may still be forced upon the media giants of old while empowering and enriching the global community that participates in the networked sharing of information. The invention of writing and later the printing press did not stop the modes of communication that came before them. Digital media is unlikely to fully replace print any time soon, but only to enhance upon what came before.

We are moving through a transitional period from print media to a new more open and global communityoriented digital medium. However, digital media is still in its infancy and we do not know yet what era defining interface will propel the digital medium beyond the dominate interface of the last 500 years. There are still barriers to be overcome, from threats to global openness to economic forces that may reduce access locally. It will take continued action from a global community committed to furthering all of humanities access to knowledge. It will also require the continued innovation of designers, researchers, and inventors to realise a truly groundbreaking interface that builds upon but exists free of the strictures and limitations of those that came before. Through these efforts, connected digital media could have an impact not unlike Gutenberg's invention of the printing press. 


\section{REFERENCES}

Bradley, J. W. (1905) Illuminated manuscripts. London: Methuen.

Cerf, V. (2009) From the Jurassic Era. Available at: http://vimeo.com/8029123 (Accessed: 11 February 2014).

Chimero, F. (2013) 'What Screens Want'. Available at: http://frankchimero.com/what-screens-want/ (Accessed:

11 February 2014).

Do, E. (2011) 'Durrell Bishop's Marble Answering Machine' Computing Creative Design Cognition. Available at: http:/ / ccdc11.wordpress.com/2011/02/15/durrell-bishops-marble-answering-machine/ (Accessed: 11 February 2014).

InternetSocietyVideo (2010) Future of the Internet: Boutique Networks. Available at: http:/ /www.youtube.com/ watch?v=YUd8dVVXmRQ (Accessed: 11 February 2014).

InternetSocietyVideo (2010) Future of the Internet: Moats and Drawbridges. Available at: http://www.youtube.com/ watch?v=ug2_GWkbF1o (Accessed: 11 February 2014).

Jones, S. (2000) 'Manuscript Illumination in Northern Europe' Metmuseum.org. [Online]. Available at: http://www. metmuseum.org/toah/hd/manu/hd_manu.htm (Accessed: 11 February 2014).

Lord, A. B. and Lord, M. L. (1995) The singer resumes the tale. Ithaca: Cornell University Press.

Marcotte, E. (2013) The Map Is Not the Territory. Available at: http://vimeo.com/63525054 (Accessed:

11 February 2014)

MIT Technology Review. (2013) 'How to Burst the "Filter Bubble" that Protects Us from Opposing Views'. Available at: http:/ / www.technologyreview.com/view/522111/how-to-burst-the-filter-bubble-that-protects-us-from-opposing-views / (Accessed: 11 February 2014).

Nagy, J. F. (1988) 'Oral Life and Literary Death in Medieval Irish Tradition', Oral Tradition, 3, pp. 368-80.

Nagy, J. F. (1986) 'Orality in Medieval Irish Narrative: An Overview', Oral Tradition, 1 (2), pp. 272-301.

Pariser, E. (2011) Beware online "filter bubbles". Available at: http://vimeo.com/21422747 (Accessed: 11 February 2014).

Victor, B. (2011) 'A Brief Rant on the Future of Interaction Design', Worrydream.com. Available at: http://worrydream. com/ABriefRantOnTheFutureOfInteractionDesign/ (Accessed: 11 February 2014).

\section{IMAGE LIST}

FIGURE 1: InternetSocietyVideo (2010) Future of the Internet: Boutique Networks. Available at: http://www.youtube.com/watch?v=YUd8dVVXmRQ (Accessed: 11 February 2014).

FIGURE 2: InternetSocietyVideo (2010) Future of the Internet: Moats and Drawbridges. Available at: http://www.youtube.com/watch?v=ug2_GWkbF1o (Accessed: 11 February 2014).

FIGURE 3: Pariser, E. (2011) Beware online "filter bubbles". Available at: http://vimeo.com/21422747 (Accessed: 11 February 2014).

FIGURE 4: Do, E. (2011) 'Durrell Bishop's Marble Answering Machine' Computing Creative Design Cognition. Available at: http://ccdc11.wordpress.com/2011/02/15/durrell-bishops-marble-answering-machine/ (Accessed: 11 February 2014).

FIGURE 5: Marcotte, E. (2013) The Map Is Not the Territory. Available at: http:/ /vimeo.com/63525054 (Accessed: 11 February 2014).

FIGURE 6: Cerf, V. (2009) From the Jurassic Era. Available at: http://vimeo.com/8029123 (Accessed: 11 February 2014).

FIGURE 7: The Young Turks. (2013) The Young Turks. Available at: https://www.youtube.com/watch?v=GPzkvmeUmsU (Accessed: 11February 2014).

FIGURE 8: The Guardian. (2012) Three Little Pigs Advert. Available at: https://www.youtube.com/watch?v=vDGrfhJH1P4 (Accessed: 11 February 2014).

FIGURE 9: Cizek, K. (2013) 'A Short History of the Highrise', The New York Times. Available at: http://www.nytimes.com/projects/2013/high-rise/ (Accessed: 11 February 2014).

FIGURE 10: Shinoda Lab, University of Tokyo. (2009) Touchable Holography. Available at: https://www.youtube.com/ watch?v=Y-P1zZAcPuw (Accessed: 11 February 2014) 